\title{
Skin toxicity in a patient with ovarian cancer treated with pegylated liposomal doxorubicin: A case report and review of the literature
}

\author{
JOANNA KUBICKA-WOŁKOWSKA, MAGDALENA KĘDZIERSKA, MAJA LISIK-HABIB and PIOTR POTEMSKI
}

Department of Chemotherapy, Medical University of Lodz, Copernicus Memorial Hospital, Lodz 93-513, Poland

Received April 16, 2015; Accepted July 15, 2016

DOI: $10.3892 / 01.2016 .5309$

\begin{abstract}
Pegylated liposomal doxorubicin (PLD) is a form of doxorubicin enclosed in pegylated liposomes. In contrast to conventional doxorubicin, PLD is characterized by a lower incidence of cardiotoxicity and myelosuppression. However, it induces specific mucocutaneous side effects, particularly palmar-plantar erythrodysesthesia (PPE). Other dermal manifestations, such as intertrigo-like dermatitis, diffuse follicular rash, melanotic macules, maculopapular rash or recall phenomenon are less common. Mechanisms that lead to skin toxicity remain unclear, however, certain reports indicate that drug excretion in sweat, host-vs.-altered-host reactions and local mechanical microtrauma play an important role in the development of cutaneous disorders. Effective preventive and curative management has not yet been established. The current study reports a case of a 55-year-old patient with advanced ovarian cancer who developed an uncommon diffuse maculopapular rash and severe PPE during treatment with PLD. Complete regression of the skin disorder was observed after 4 weeks. At present, palliative chemotherapy provides the opportunity to prolong life and alleviate disease symptoms, nonetheless it produces a number of adverse effects. Dermal complications may affect patient quality of life and cause therapy interruption. In the light of widespread use of PLD, skin toxicity associated with this drug creates a major problem.
\end{abstract}

\section{Introduction}

Conventional doxorubicin is used actively in various malignant tumors, however, it produces a number of serious side effects, including cardiotoxicity and myelosuppression (1). A new form of this chemotherapeutic agent enclosed in pegylated

Correspondence to: Mrs. Joanna Kubicka-Wołkowska, Department of Chemotherapy, Medical University of Lodz, Copernicus Memorial Hospital, U1. Pabianicka 62, Lodz 93-513, Poland

E-mail: zuz.ka@interia.pl

Key words: skin toxicity, pegylated liposomal doxorubicin, maculopapular rash, ovarian cancer liposomes was developed to reduce these organ toxicities (1). Liposome encapsulation prevents doxorubicin from penetration to compartments with tight endothelial cells junctions and facilitates its distribution to tissues with abnormal blood vessels (1). This results in higher drug accumulation within the tumor when compared with normal tissues (2). Consequently, a decreased incidence of cardiac and hematological toxicity is observed. Pegylated liposomal doxorubicin (PLD) has the ability to deposit itself within the skin and to induce specific mucocutaneous reactions. There are six types of PLD-related dermal disorders, and the most common is palmar-plantar erythrodysesthesia (PPE). Other, less frequent manifestations are intertrigo-like dermatitis, a diffuse follicular rash, a maculopapular rash, melanotic macules or a recall phenomenon (3). The symptoms of PPE develop usually within 2 to 12 days after the infusion of chemotherapy (4). Initially, dysesthesia, erythema or edema of the palms and plantae is noticed. These symptoms may progress to desquamation, blistering and ulceration. The soles are less often affected than the palms (5).

The current study presents the case of a patient with advanced ovarian cancer treated with PLD who developed severe hand-foot syndrome and a diffuse maculopapular rash, which is rarely reported in the literature. Complete resolution of the skin lesions was observed after 4 weeks. Due to ovarian cancer progression, the patient was disqualified from further chemotherapy.

\section{Case report}

A 55-year-old patient without any relevant medical history underwent suboptimal cytoreductive surgery involving a hysterectomy, bilateral salpingo-oophorectomy, omentectomy and appendectomy in November 7, 2011 at the Polish Mother's Memorial Hospital Research Institute (Lodz, Poland), and was accordingly diagnosed with stage IIIC ovarian cancer based on the tumor-node-metastasis classification criteria (6). The patient received 6 cycles of intravenous paclitaxel $(175 \mathrm{mg} /$ $\mathrm{m}^{2}$ ) and carboplatin [area under the curve (AUC), 5], administered every 3 weeks, and then follow-up surgery with cervical amputation. The disease was considered to be in complete remission until November 2012, when rapid progression with accompanying intestinal obstruction was observed. The patient underwent ileostomy formation, and due to significant loss in body weight, started parenteral nutrition. Subsequently, 
from January to June 2013, 2 cycles of cisplatin $\left(70 \mathrm{mg} / \mathrm{m}^{2}\right.$ every 3 weeks) were administered, followed by 6 cycles of carboplatin (AUC, 5). Cisplatin was discontinued due to renal insufficiency. A partial response to chemotherapy was observed. During this time, no skin toxicity was noted. When the ovarian cancer progressed again, therapy with $50 \mathrm{mg} / \mathrm{m}^{2}$ PLD administered every 4 weeks was initiated. No prevention strategies for PPE were implemented. At 3 weeks after the second cycle of chemotherapy, the patient developed a rash localized on the trunk and severe skin lesions on the hands. Dermatological evaluation revealed painful desquamative erythema with ulceration on the palms (Fig. 1) and mild erythema on the soles. Non-pruriginous, non-painful maculopapular eruption accompanied by peeling was present on the trunk (Fig. 2). Oral mucous membranes and other areas of the skin were not affected. The patient was classified with grade 3 PPE (according to the basic scale from the Common Terminology Criteria for Adverse Events, version 4), as difficulties were exhibited in self-care activities, and a grade 3 maculopapular rash (7). Upon admission on October 25, 2013, the patient was apyretic and in a good general condition (Eastern Cooperative Oncology Group performance status 2), with the main complaint being of pain due to ulcerative cutaneous lesions. No previous episodes of drug allergies were reported. The patient started $100 \mathrm{mg}$ tramadol and $100 \mathrm{mg}$ doxycycline, administered twice daily, and prophylactic antifungal treatment with $50 \mathrm{mg}$ fluconazole, administered daily. Amelioration of the skin lesions was observed after 5 days of therapy, and complete regression was apparent after 4 weeks. In November 2013, there was a sudden deterioration in the patient's general condition. Follow-up abdominal ultrasound and laboratory blood tests [carbohydrate antigen-125, 4,495 U/ml (normal range, $<35 \mathrm{U} / \mathrm{ml}$ ); and bilirubin, $4.3 \mathrm{mg} / \mathrm{dl}$ (normal range, $0.3-1.2 \mathrm{mg} / \mathrm{dl}$ )] revealed dynamic progression of the malignancy. The patient was therefore disqualified from further chemotherapy and referred to a palliative care specialist. The patient succumbed to cancer progression in December 2013.

\section{Discussion}

Dermal toxicity is the most common adverse reaction limiting PLD therapy. Skin lesions usually appear in regions prone to trauma, such as the palms and soles. PPE of any grade is observed in up to $50 \%$ of individuals treated with PLD, while grade 3 is noted in $\sim 20 \%$ of patients (when using a PLD dose of $50 \mathrm{mg} / \mathrm{m}^{2}$ every 4 weeks) (8). Less frequently intertriginous areas, such as axillary folds, are affected. The maculopapular rash present in the current patient has rarely been reported in the literature (9-11).

The pathophysiology of this cutaneous syndrome is widely debated. It is presumed that drug excretion in sweat and local microtrauma are responsible for the development of PPE (12). Certain data have indicated that PLD may penetrate through the damaged vessels and impair keratinocytes, which are particularly susceptible to anticancer drugs (13). An elevated PLD concentration found in the skin of the palms and plantae supports the hypothesis that the chemotherapeutic agent is excreted in the sweat. Jacobi et al (14) reported the appearance of PPE only in patients with hyperhidrosis of these regions. Another hypothesis is that PPE develops due to an excessive concentration of

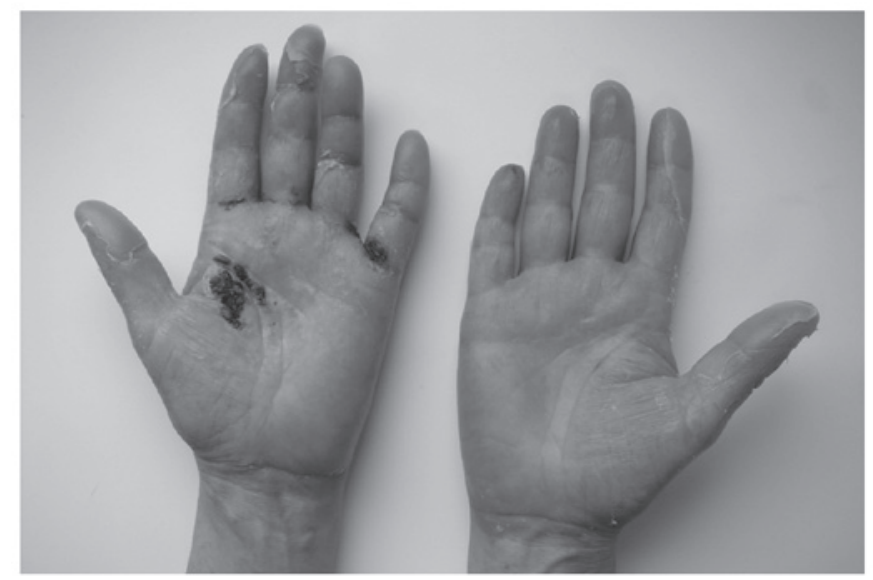

Figure 1. Clinical manifestation of palmar-plantar erytrodysesthesia: Erythema, desquamation and ulceration on the palmar sides of the hands.

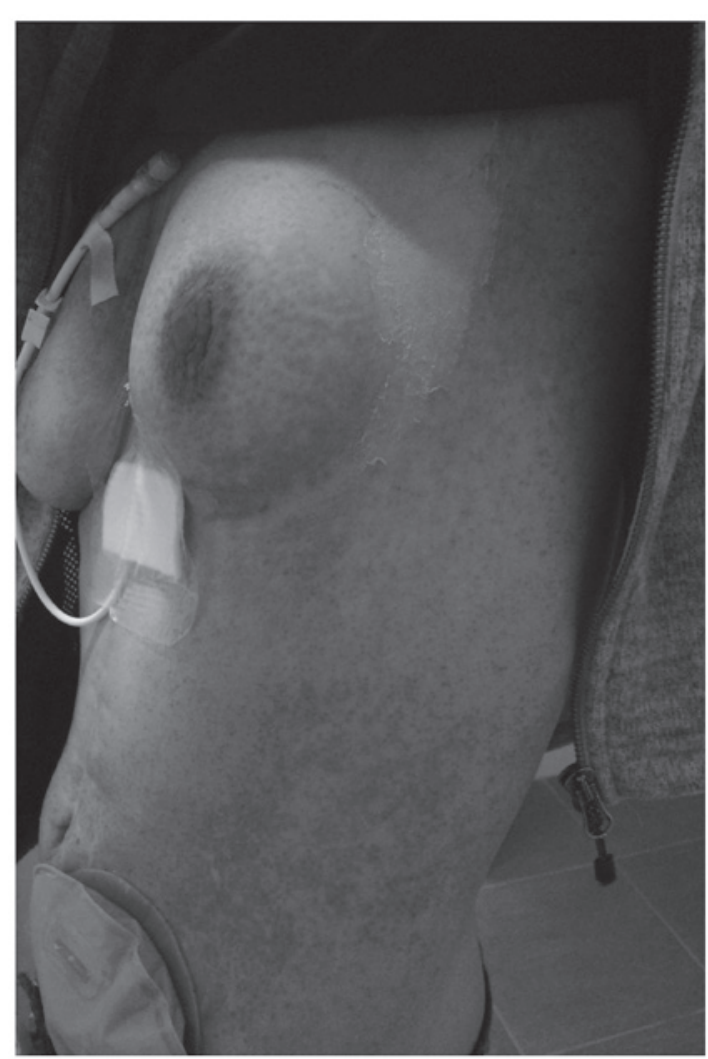

Figure 2. Clinical appearance: Maculopapular rash on the trunk.

toxic doxorubicin within the skin and its reaction with metal ions (particularly copper ions) (15). An underlying mechanism for the development of other skin disorders is poorly known. Skelton et al (9), on the basis of the late outbreak of dermal lesions and lymphocytic inflammation affecting keratinocytes found in the skin biopsies of 3 patients with PLD-induced maculopapular rash, suggested the possibility of host-vs.-altered-host reaction as a key factor responsible for the development of cutaneous syndromes. Optimal management of PLD-related skin reactions remains undefined. It may appear that numerous clinical trials have been performed, but in fact, the majority of them have limited value (5). Preventive approaches for PPE, including administration of moisturizers, regional cooling of 
the skin, and avoidance of excessive activities associated with overheating or trauma, have been evaluated in non-randomized trials $(4,16)$. The use of topical antiperspirant with a beneficial effect has also been reported in the literature (17). Pyridoxine appeared to be a promising agent for the prevention of PPE, but in randomized controlled trials, it proved to be ineffective $(18,19)$. In a meta-analysis conducted by Macedo et al (16), celecoxib exclusively demonstrated a $53 \%$ risk reduction (odds ratio, $0.47 ; 95 \%$ confidence interval, $0.29-0.78 ; \mathrm{P}=0.003$ ) of any grade PPE. Certain studies have indicated that dimethyl sulfoxide $(20)$ or corticosteroids $(21,22)$ may be beneficial in the treatment of PLD-induced dermal complications, as they accelerate skin recovery, but in fact, the only well-established preventive management includes dose intensity modification or complete chemotherapy discontinuation (11).

In conclusion, apart from PPE, other skin toxicities associated with PLD treatment are less frequent and not well known. The aforementioned prophylactic and curative strategies for PLD-induced dermal toxicity require further investigation, and their usage in routine clinical practice is unsupported. As mucocutaneous side effects are an important cause of PLD dose modification or treatment withdrawal, it is essential to conduct prospective randomized controlled clinical trials in order to strictly define the preventive and curative management of this complication.

\section{Acknowledgements}

This study was supported by a grant from the Chemotherapy Clinic of the Medical University of Lodz (no. UM 501/1-034-02/501-91-263).

\section{References}

1. Mangana J, Zipser MC, Conrad C, Oberholzer PA, Cozzio A, Knuth A, French LE and Dummer R. Skin problems associated with pegylated liposomal doxorubicin - more than palmoplantar erythrodysesthesia syndrome. Eur J Dermatol 18: 566-570, 2008

2. Green AE and Rose PG: Pegylated liposomal doxorubicin in ovarian cancer. Int J Nanomedicine 1: 229-239, 2006.

3. Cady FM, Kneuper-Hall R and Metcalf JS: Histologic patterns of polyethylene glycol-liposomal doxorubicin-related cutaneous eruptions. Am J Dermatopathol 28: 168-172, 2006.

4. Farr KP and Safwat A: Palmar-plantar erythrodysesthesia associated with chemotherapy and its treatment. Case Rep Oncol 4: 229-235, 2011

5. von Moos R, Thuerlimann BJ, Aapro M, Rayson D, Harrold K, Sehouli J, Scotte F, Lorusso D, Dummer R, Lacouture ME, et al: Pegylated liposomal doxorubicin-associated hand-foot syndrome: Recommendations of an international panel of experts. Eur J Cancer 44: 781-790, 2008.

6. Sobin LH, Gospodarowicz MK and Wittekind C (eds): Ovary cancer. In: TNM Classification of Malignant Tumours. 7th edition. Wiley-Blackwell, Hoboken, NJ, pp222-226, 2009.
7. National Cancer Institute: Common Terminology Criteria for Adverse Events (CTCAE) version 4.03. http://evs.nci.nih.gov/ ftp1/CTCAE/CTCAE 4.03_2010-06-14_QuickReference_5x7. pdf. Accessed June 14, 2010.

8. Lorusso D, Di Stefano A, Carone V, Fagotti A, Pisconti S and Scambia G: Pegylated liposomal doxorubicin-related palmar-plantar erythrodysesthesia ('hand-foot' syndrome). Ann Oncol 18: 1159-1164, 2007.

9. Skelton H, Linstrum J and Smith K: Host-vs.-altered-host eruptions in patients on liposomal doxorubicin. J Cutan Pathol 29: 148-153, 2002.

10. English JC III, Toney R and Patterson JW: Intertriginous epidermal dysmaturation from pegylated liposomal doxorubicin J Cutan Pathol 30: 591-595, 2003.

11. Vidal C, Afonzo Y, Abal C and Rondón M: A grade IV maculopapular skin lesion associated with pegylated liposomal doxorubicin. MOJ 2: 57-59, 2012.

12. Martschick A, Sehouli J, Patzelt A, Richter H, Jacobi U, Oskay-Ozcelik G, Sterry W and Lademann J: The pathogenetic mechanism of anthracycline-induced palmar-plantar erythrodysesthesia. Anticancer Res 29: 2307-2313, 2009.

13. Kim RJ, Peterson G, Kulp B, Zanotti KM and Markman M: Skin toxicity associated with pegylated liposomal doxorubicin $(40 \mathrm{mg} / \mathrm{m} 2)$ in the treatment of gynecologic cancers. Gynecol Oncol 97: 374-378, 2005.

14. Jacobi U, Waibler E, Schulze P, Sehouli J, Oskay-Ozcelik G, Schmook T, Sterry W and Lademann J: Release of doxorubicin in sweat: First step to induce the palmar-plantar erythrodysesthesia syndrome? Ann Oncol 16: 1210-1211, 2005.

15. Yokomichi N, Nagasawa T, Coler-Reilly A, Suzuki H, Kubota Y, Yoshioka R, Tozawa A, Suzuki N and Yamaguchi Y: Pathogenesis of hand-foot syndrome induced by PEG-modified liposomal doxorubicin. Hum Cell 26: 8-18, 2013.

16. Macedo LT, Lima JP, dos Santos LV and Sasse AD: Prevention strategies for chemotherapy-induced hand-foot syndrome: A systematic review and meta-analysis of prospective randomised trials. Support Care Cancer 22: 1585-1593, 2014.

17. Templeton AJ, Ribi K, Surber C, Sun H, Hsu Schmitz SF Beyeler M, Dietrich D, Borner M, Winkler A, Müller A, et al: Prevention of palmar-plantar erythrodysesthesia with an antiperspirant in breast cancer patients treated with pegylated liposomal doxorubicin (SAKK 92/08). Breast 23: 244-249, 2014.

18. Kang YK, Lee SS, Yoon DH, Lee SY, Chun YJ, Kim MS, Ryu MH, Chang HM, Lee JL and Kim TW: Pyridoxine is not effective to prevent hand-foot syndrome associated with capecitabine therapy: Results of a randomized, double-blind, placebo-controlled study. J Clin Oncol 28: 3824-3829, 2010.

19. von Gruenigen V, Frasure H, Fusco N, DeBernardo R, Eldermire E, Eaton $\mathrm{S}$ and Waggoner S: A double-blind, randomized trial of pyridoxine versus placebo for the prevention of pegylated liposomal doxorubicin-related hand-foot syndrome in gynecologic oncology patients. Cancer 116: 4735-4743, 2010.

20. Lopez AM, Wallace L, Dorr RT, Koff M, Hersh EM and Alberts DS: Topical DMSO treatment for pegylated liposomal doxorubicin-induced palmar-plantar erythrodysesthesia. Cancer Chemother Pharmacol 44: 303-306, 1999.

21. Drake RD, Lin WM, King M, Farrar D, Miller DS and Coleman RL: Oral dexamethasone attenuates Doxil-induced palmar-plantar erythrodysesthesias in patients with recurrent gynecologic malignancies. Gynecol Oncol 94: 320-324, 2004.

22. Najem A, Deregnaucourt D, Ramdane S, Dridba M, Djouba F and Vercambre-Darras S: Intertrigo-like dermatitis with pegylated liposomal doxorubicin: Diagnosis and management. J Clin Oncol 32: e104-e106, 2014. 